\title{
Digitale Gesundheit: Was ändert sich für den Gesundheitsbegriff?
}

\author{
Bernhard Wieser
}

(C) Der/die Autor(en) 2019

Zusammenfassung Dieser Beitrag untersucht, wie sich mit der Entwicklung digitaler Gesundheitstechnologien das Verständnis von Krankheit und Gesundheit ändert. Digitale Technologien sind in einem bedeutsamen Sinn transgressiv. Sie gehen über die kurative und präventive Medizin hinaus und überschreiten die Grenzen zu einer wunscherfüllenden Medizin, die nach Verbesserung und Optimierung des menschlichen Körpers und seiner Leistungsfähigkeit sucht. Vor allem ist digitale Medizin eine Vision. Nicht, was es bereits gibt, sondern was möglich sein könnte, wird mit diesem Begriff in Verbindung gebracht. Digitale Medizin gibt ein Versprechen ab: Wenn es gelingt, medizinisches Wissen und gesundheitsbezogene Daten umfassend zu integrieren, wird es möglich, Krankheiten besser zu heilen, ja sogar ihr Auftreten $\mathrm{zu}$ verhindern. In Form eines medizinischen Avatars gießt sich diese Vision in eine konkrete technologische Gestalt. Ein solcher digitaler Zwilling stellt ein Abbild aller physiologischen und psychischen Vorgänge bereit, auf dessen Basis gesundheitsbezogene Prozessabläufe simuliert und therapeutische Maßnahmen evaluiert werden können. Aufgabe sozialwissenschaftlicher Reflexion ist es nun, im Sinne eines $V i$ sion Assessments die Implikationen neuer Technologien zu diskutieren, bevor sie Realität geworden sind. Besonders bedeutsam sind im diskutierten Zusammenhang die technologisch vermittelten Möglichkeiten eines lückenlosen Monitorings und einer umfassenden Simulation. Durch digitale Gesundheitstechnologien ändert sich nicht nur der Interventionsmodus medizinischen Handelns, sondern auch das, was wir autonomes Subjekt nennen. Gesundheit wird zu einer quantifizierbaren Zielvorgabe und zu einer Optimierungspraxis. Letztlich befördert dies die Ökonomisierung des Sozialen und verändert in tiefgreifender Weise, wie wir uns verhalten, um unsere Gesundheit zu erhalten, Krankheiten heilen oder präventiv verhindern.

\footnotetext{
B. Wieser $(\bowtie)$

Institute of Interactive Systems and Data Science, Graz University of Technology, Inffeldgasse 16c, $8010 \mathrm{Graz}$, Österreich

E-Mail: bernhard.wieser@tugraz.at
} 
Schlüsselwörter Digitale Medizin, · E-Health · Simulation · Avatar · Cyborgs · Vision Assessment · Gesundheit

\title{
Digital Health: what is changing for our understanding of health?
}

\begin{abstract}
In this paper, I discuss how the development of digital health technologies changes our understanding of health and illness. Digital technologies are transgressive in important ways: They go beyond curative and preventive medicine and cross over to a kind of medicine that caters to desires of enhancement and optimisation of the human body. Digital medicine is, first and foremost, a vision: it refers not only to existing technologies, but also to prospective ones. Digital medicine makes a promise: If we succeed with integrating medical knowledge and health related data in a comprehensive way, better therapies and the prevention of illness will be possible. One of the manifestations of this vision are medical avatars. Such digital twins are virtual representations of physiological and psychological conditions of patients and provide a basis for a simulation of health-related processes and the evaluation of therapeutic measures. Using vision assessment as a method, it is the task of sociological analysis to discuss the wider implications of emerging technologies before they materialise. It is of particular interest to study digital health technology as enabler of a comprehensive monitoring and simulation as well as the ways in which they change the mode of intervention in medical practice. Another thing that digital health technologies are changing are traditional conceptions of autonomous subjects. Health becomes a quantifiable target and a practice of optimisation. This, in turn, promotes the economisation of the social, and changes what we do in order to maintain our health and cure or prevent diseases in fundamental ways.
\end{abstract}

Keywords Digital medicine $\cdot$ E-health $\cdot$ Simulation $\cdot$ Avatar $\cdot$ Cyborgs $\cdot$ Vision assessment $\cdot$ Health

\section{Einleitung}

Die Entwicklung neuer Technologien lädt ein, über die Frage nachzudenken, wie sich unsere Welt mit ihrer Diffusion verändert. Nicht nur, aber gerade auch im Bereich der Medizin stellen sich solche Fragen. Sozialwissenschaftliche Reflexion versucht Antworten darauf zu geben, (ob und) wie sich medizinisches Handeln ändert, wenn neue Technologien angewandt werden. Bis vor geraumer Zeit wurde diese Reflexion über moderne Medizin von den Entwicklungen in der Genomforschung dominiert (z. B. Lemke 2000, 2003, 2004a, 2007, 2011; Habermas 2001; Lippman 1991, 1994; Novas und Rose 2000; Rose 1999; Rose und Novas 2005; Rose 2007; Rheinberger 1997, 1999; Rheinberger et al. 1997). Doch zunehmend gerät das Thema Digitalisierung ins Zentrum der Aufmerksamkeit. Die Nutzung medizinischer Daten und ihre informationstechnische Auswertung und Verarbeitung versprechen ein vertieftes Verständnis von Krankheit und, darauf aufbauend, die Möglichkeit zur Prävention (vgl. Hood 2013). Der vorliegende Text nimmt diese Entwicklungen zum Anlass und versucht, Antworten auf die Frage zu finden, in welcher Weise sich mit 
digitalen Technologien ändert, wie wir Krankheit und Gesundheit verstehen, und was wir tun können, um Krankheiten zu behandeln sowie Gesundheit zu erhalten und zu fördern.

Was zur Wiederherstellung, Erhaltung und Förderung von Gesundheit getan werden kann, schließt ärztliches Handeln ein, geht aber weit darüber hinaus. Digitale Technologien sind in einem bedeutsamen Sinn transgressiv, sie gehen über die kurative und präventive Medizin hinaus und überschreiten die Grenzen zu einer wunscherfüllenden Medizin, die nach Verbesserung und Optimierung des menschlichen Körpers und seiner Leistungsfähigkeit sucht. Gerade das Aufweichen dieser Grenzen sehe ich im Zusammenhang damit, was digitale Medizin genannt werden kann. Bewusst wird der Anwendungskontext digitaler Medizin über ärztliches Handeln hinausgedacht, zumal ihre Technologien auch von den Individuen selbst angewandt werden können und nicht nur auf den diagnostischen oder kurativen Kontext beschränkt sind, sondern auch im Fitness- und Lifestyle-Bereich eingesetzt werden. Nikolas Rose formuliert: ,The old lines between treatment, correction, and enhancement can no longer be sustained" (Rose 2007, S. 6; siehe auch Lupton 2013, 2016; Lupton und Jutel 2015; Petersen und Lupton 1996; Selke 2014; Duttweiler et al. 2016; Grasse und Greiner 2013). ${ }^{1}$

Die Anwendungsmöglichkeiten digitaler Technologien im Gesundheitsbereich sind derart mannigfaltig, dass es schwerfällt, eine vollständige Analyse aller Bereiche vorzunehmen. Im medizinischen Kontext umfasst der Einsatz digitaler Technologien beginnend bei der elektronischen Erfassung und Verwaltung von Patientenakten, Erstellung, Weitergabe und Archivierung von Befunden, Untersuchungsdaten, Rezepten und Therapieplänen bis hin zum Bereich der Kostenrechnung und Rückverrechnung mit Versicherungen unterschiedlichste Teilbereiche. Digitale Technologien sind Bestandteil diagnostischer Geräte, und sie werden für Modellierungen und Simulationen eingesetzt. Im vorliegenden Text wird es weniger um medizinische Forschung oder administrative Anwendung digitaler Technologien gehen, sondern hauptsächlich um jene Anwendungen, die in einer interaktiven Beziehung zu menschlichem (Gesundheits-)Verhalten stehen, dieses zu erfassen und zu steuern suchen. Im Vordergrund stehen damit Interface-Technologien (insbesondere digitale Avatare), mit welchen diese Beziehung zwischen Menschen und computerbasierten Informationssystemen technisch realisiert werden sollen. Zum Teil werden solche Technologien bereits eingesetzt. Fitnessuhren (Wearables) sind ein anschauliches Beispiel dafür. Die hier vorgenommene Betrachtung greift gegenwärtige Technologien auf, geht jedoch über solche, bereits existierenden Anwendungen hinaus und bezieht Entwicklungsziele ein, die noch nicht erreicht oder umgesetzt sind. Das Öffnen der Analyseperspektive auf die Zukunft ist wichtig, wenn die Gestaltung künftiger Technologien nicht als alleinige Aufgabe von IngenieurInnen², sondern

\footnotetext{
1 Zur synonymen Bedeutung von ,health“ und „wellness“ siehe insbesondere Diaz et al. (2013); Flores et al. (2013); Kirschner et al. (2013).

2 Zur bedeutsamen Rolle von MathematikerInnen, PhysikerInnen, Computer Scientists und IngenieurInnen als maßgebliche AkteurInnen der digitalen Medizin vergleiche insbesondere Anthony et al. (2012); Hunter et al. (2013); Wolkenhauer et al. (2013); Wang et al. (2015). Sowie insbesondere Moreno et al. 2011.
} 
als gesellschaftliches Projekt verstanden wird, zu dem auch sozialwissenschaftliche Reflexion beitragen kann.

\section{Methodisches Vorgehen}

Medizinische Avatare werden als computerbasierte Technologien verstanden, die dazu in der Lage sind, physiologische, psychische und soziale Abläufe (vollständig) zu erfassen und zu prozessieren (Hunter et al. 2013). Ein solcher ,digitaler Zwilling“ ist jedoch nicht bloß ein Abbild - ein gläserner Mensch, sondern eine Simulation. Der Unterschied ist wesentlich. Er liegt darin, dass mit einer Simulation Prozessabläufe (prädiktive Experimente) getestet werden können. Auf diese Weise kann erprobt werden, wie sich die Veränderung von ausgewählten Parametern auf das simulierte System auswirkt. So könnte etwa geklärt werden, welches von zwei Medikamenten für eine bestimmte Person wirksamer ist. Die Idee eines solchen medizinischen Avatars bringt die Möglichkeiten der digitalen Medizin auf den Punkt (vgl. Vogt et al. 2016, S. 136). Diese Konkretisierung erlaubt es mir, die Reflexion über die Implikationen der Verwendung digitaler Technologien im Bereich der Wiederherstellung, Erhaltung und Förderung von Gesundheit greifbar und damit auch diskutierbar zu machen. Einen omnipotenten Avatar, wie er hier angesprochen wird, gibt es noch nicht. Es handelt sich vielmehr um ein Innovationsziel. Im Projekt „Modelling Health“, gefördert aus Mitteln des Zukunftsfonds Steiermark ${ }^{3}$ haben ExpertInnen aus Medizin, Informatik und Pflegewissenschaft gemeinsam an der Ausarbeitung dieses Innovationsziels gearbeitet. Die Aufgabe des Autors im Kontext dieses Projekts war es, die gesellschaftliche Wünschbarkeit medizinischer Avatare zu erörtern.

Aus methodischer Sicht ist es keinesfalls einfach, einen Problemgegenstand zu untersuchen, dessen Anwendungspraxis in der Zukunft liegt. AnwenderInnen können nicht zu ihren Erfahrungen befragt werden. Gegenstand der Analyse sind vielmehr Vorstellungen, Perspektiven und Ziele. Daher habe ich mich im vorliegenden Text an der Methode des Vision-Assessment orientiert (vgl. Lösch 2013). Die Idee dieser Vorgehensweise besteht darin, die Implikationen neu entstehender Technologien zu diskutieren, bevor sie Realität geworden sind. Um der Frage vorzugreifen, warum man das überhaupt tun sollte, verweise ich darauf, was in der Technikfolgenabschätzung das „Collingridge Dilemma“ (Collingridge 1980; Grunwald 2010) genannt wird. Collingridge weist darauf hin, dass man nicht sagen kann, wie sich eine Technologie auswirken wird, bevor sie angewandt wird. Wartet man aber so lange, bis dies der Fall ist, sind viele Entscheidungen bereits getroffen, und damit wird es sehr schwer, steuernd einzugreifen, um die Technologien so zu gestalten,

\footnotetext{
3 Der vorliegende Text ist aus einem Beitrag zum Expertenworkshop „Transformation von Medizin, Pflege, Krankheit und Institutionen: Big Data im Gesundheitswesen“ entstanden. Ich wurde vom Institut für Technikfolgenabschätzung und Systemanalyse (ITAS) am Karlsruher Institut für Technologie (KIT) eingeladen, über die Transformation des Gesundheitsbegriffs im Kontext von Big Data zu sprechen. Grund für die Einladung war meine Mitarbeit im Forschungsprojekt „Modelling Health“ (gefördert aus Mitteln des Zukunftsfonds Steiermark). In diesem Projekt - das für den vorliegenden Text ein wichtiger Bezugspunkt ist - wurde die Wünschbarkeit von medizinischen Avataren untersucht.
} 
dass sie dem entsprechen, was in einer Gesellschaft für wünschenswert gehalten wird.

Mit diesem Beitrag wird versucht, die Reflexion frühzeitig zu beginnen. Der Weg, der dafür gewählt wurde, besteht darin, sich eine Technologie vorzustellen, die all das konkretisiert, was man unter dem Sammelbegriff digitale Medizin fassen kann (vgl. Lupton 2016). Zur Diskussion steht demnach eine Technologie, die in der Lage ist, ein umfassendes Monitoring durchzuführen, die Menschen zu jeder Zeit und an jedem Ort begleitet, sämtliche medizinischen Daten erhebt und zur Weiterverarbeitung bereitstellt. Sensoren am Körper, in der Kleidung und im (häuslichen) Umfeld generieren Daten, die von digitalen Technologien gesammelt und prozessiert werden. Doch obwohl eine vollständige Simulation der physiologischen, psychologischen und sozialen Prozesse derzeit noch nicht möglich ist, so sind medizinische Avatare, die dazu in der Lage sind, keine völlige Spekulation. Tatsächlich arbeiten viele Forschungsteams rund um den Globus an seiner technischen Realisierung (vgl. Diaz et al. 2013, S. 57; Hunter et al. 2013; Vogt et al. 2016, S. 316). Eine konkrete Artikulation dieser Vision findet sich etwa in der Roadmap der Europäischen Kommission für den „Digital Patient“, wo es heißt:

The vision of a "digital me" that contains all my health-care information, [...] communicated with all my wearable and implanted technology to constantly monitor my health status and informing me, my family and friends, or my healthcare providers of alarming events, supporting the collaboration of various specialists around my complex systemic diseases, and used with all my data to predict the future development of my health in order to facilitate disease prevention and a fully self-aware lifestyle. (Diaz et al. 2013, S. 57)

Vor diesem Hintergrund ist eine Auseinandersetzung mit Technologien, die in Entwicklung sind, mit Visionen, die solche Innovationsvorhaben inspirieren, und mit gesellschaftspolitischen Rahmenbedingungen, die den Bedarf dafür konstituieren, eine aktuelle Aufgabe sozialwissenschaftlicher Reflexion.

\section{Die Handlungsträgerschaft von Technik}

Wesentlich für den hier gewählten methodischen Zugang ist es, die zur Diskussion gestellten Implikationen nicht als zwingende Folgen zu verstehen. Vielmehr geht es im Sinne des schon angesprochenen Vision-Assessment darum, zu fragen, ob wir es für wünschenswert halten, wenn es so kommt. Der Unterschied ist wesentlich und erklärt sich aus der Auseinandersetzung mit dem Zusammenwirken von sozialem und technischem Wandel. Sprachlich sind wir dazu geneigt, den analysierten Technologien Handlungsfähigkeit zuzuschreiben. Dadurch entsteht der Eindruck, dass sie es sind, welche die erörterten Veränderungen ursächlich herbeiführen würden oder gar Motor der Geschichte wären. Solche verkürzten Darstellungen werden in der sozialwissenschaftlichen Technikforschung „Technik-Determinismus“ genannt (vgl. Pinch und Bijker 1984; Smith und Marx 1994; Wyatt 2008). Kritisiert werden mit diesem Begriff Erklärungen, die gesellschaftliche Entwicklungen als exklusive Folge technologischer Innovationen darstellen. Ingo Schulz-Schaeffer spricht diesbezüg- 
lich von Konsequentialismus (vgl. Schulz-Schaeffer 2000). Problematisch werden solche Erklärungen, wenn sie monokausal, unidirektional und alternativenlos sind. Gefordert werden vor diesem Hintergrund Zugänge, die sozialen und technischen Wandel als zusammengehörig analysieren und nicht das eine als die Ursache des anderen betrachten. Sheila Jasanoff schlägt in diesem Sinne vor, über Wechselseitigkeit hinausgehend und von „Co-Production“ zu sprechen (vgl. Jasanoff 2004, 2005). Soziotechnischer Wandel ist vor diesem Hintergrund sicherlich nicht auf singuläre Faktoren zu reduzieren. Insbesondere haben VertreterInnen der Akteur-NetzwerkTheorie versucht, der Verbindung von technischen Artefakten und menschlichen AkteurInnen Rechnung zu tragen und nachdrücklich betont, dass es nicht zielführend sei, soziale Praktiken zu analysieren, ohne zu berücksichtigen, welche Technologien dabei im Spiel sind (Latour 1994, 2000). Latour hebt hervor, dass die Verbindung von Menschen und Technik Möglichkeiten eröffnet, neue Handlungen zu vollziehen. Technologien handeln also nicht losgelöst von menschlichen AkteurInnen, sondern sie sind vielmehr Ermöglicher (enabler) von Handlungen, die weder Mensch noch Technik alleine vollziehen könnte. Ich trage diesen Überlegungen Rechnung, indem ich formuliere, was sich MIT dem Einsatz digitaler Interface-Technologien ändert (nämlich die Eröffnung von Handlungs-Möglichkeiten) und nicht DURCH sie. Ein weiteres Anliegen techniksoziologischer Literatur richtet den Fokus auf das Design von Technologien und unterstreicht auch hier die Möglichkeit alternativer Entwicklungspfade (vgl. Bijker 2010). Dieses Postulat richtet sich gegen die Vorstellung, dass technische Entwicklungen einer Eigenlogik folgten, die sich losgelöst von sozialen Einflüssen vollzöge. Mit dem Aufzeigen von alternativen Entwicklungspfaden öffnet sich die Perspektive auf die Gestaltbarkeit von Technologien und ihrer Anwendungspraktiken (vgl. Bijker und Law 1992). Der vorliegende Beitrag schließt an diese Diskussion an. Das von mir gezeichnete Bild ist somit keine Prognose, sondern es ist ein Szenario, das auf seine Wünschbarkeit befragt werden kann.

\section{Roadmap}

Zunächst werde ich zusammenfassen, was hier mit dem Begriff ,digitale Medizin“ gemeint ist. Als Nächstes will ich erläutern, wie solche Technologien insbesondere für das Thema der Prävention maßgeblich sind. In einem dritten Schritt werde ich an die Diskussion im Kontext der medizinischen Genomforschung erinnern. Das mache ich deshalb, weil in diesem Zusammenhang sehr viele Aspekte, die auch für die digitale Medizin relevant sind, bereits auf einem hohen Reflexionsniveau diskutiert wurden. Ausgehend davon werde ich die Frage klären, ob und in welcher Weise digitale Medizin über das hinausgeht, was sich bereits im Kontext der genetischen Medizin abzuzeichnen begonnen hat.

\section{Das Versprechen der digitalen Medizin}

Digitale Technologien werden mit großen Innovationschancen in Verbindung gebracht. Dafür steht nicht zuletzt das Schlagwort „Digitalisierung“. Im medizini- 
schen Kontext wird insbesondere das Potenzial angesprochen, durch die Nutzung und computergestützte Auswertung mannigfaltiger Daten zu neuen Möglichkeiten im Umgang mit Gesundheit und Krankheit zu kommen (vgl. Kolany-Raiser et al. 2018). Auch wenn es bereits eine ganze Reihe von Technologien gibt, die gesundheitsbezogene Daten sammeln und prozessieren, wird digitalen Technologien im Gesundheitsbereich ein Potenzial zugesprochen, das noch lange nicht ausgeschöpft ist. Die Feststellung dieses großen Potenzials ist selbst ein Projekt, das von den Proponenten dieser Technologie diskursiv vorangetrieben wird. Digitalisierung ist vor diesem Hintergrund nicht nur ein Prozess, der sich empirisch beobachten lässt, sondern vor allem eine prospektive Entwicklung, die vorangetrieben werden soll. Die Soziologin Debora Lupton hebt diesen Aspekt hervor, wenn sie von ,digitaler Medizin“ als Vision spricht (Lupton 2016). Es ist diese in die Zukunft gerichtete Perspektive, die hier diskutiert werden soll. Solche Leitperspektiven haben die Funktion, Entwicklungen voranzutreiben, Ressourcen zu mobilisieren und politischen Rückhalt dafür sicherzustellen. Darüber hinaus geben sie Forschungs- und Innovationsbemühungen eine gewisse Richtung, einen Horizont, auf den hingeforscht und entwickelt wird.

Im Sinne einer Leitperspektive wird digitale Medizin zum symbolischen Bedeutungsträger eines Versprechens. Auf den Punkt gebracht lautet es: Wir können alles wissen! Wir können alles erfassen und das gesamte daraus resultierende Wissen verfügbar machen. Wenn wir nur genug Daten erfassen, können wir alle medizinischen Probleme lösen. Dann haben wir eine Antwort auf alle Fragen und können somit auch alle Krankheiten heilen, ja sogar dafür sorgen, dass sie erst gar nicht auftreten. In der englischsprachigen Literatur wird diese Vision auch unter den Begriffen ,holistic“ oder ,integrative“ beschrieben (Auffray und Hood 2012; Vandamme et al. 2013), man spricht auch von P4-Medizin im Sinne einer Praxis, die ,predictive, preventive, personalized and participatory“ ist (Hood und Flores 2012; Hood 2013). Es handelt sich um einen techno-wissenschaftlichen Holismus, der davon ausgeht, dass medizinische und insbesondere digitale Technologien alles um- und erfassen können (Vogt et al. 2016, S. 310). Vogt, Hoffmann und Getz bringen diesen Gedanken auf den Punkt: „And while the metaphor of the genome as the ,book of life " may be obsolete, and the road towards unravelling the actual mechanistic working of the body long, the whole life story may still be portrayed as information that biomedicine can decipher" (Vogt et al. 2016, S. 313-314). Wenn es gelingt, alles Wissen und alle Daten zu integrieren, soll es möglich werden, alle physiologischen, psychischen und sozialen Vorgänge abzubilden. Es ist wesentlich, dass die Vision der ,digitalen Medizin" nicht beim Erfassen, Protokollieren und Abbilden stehenbleibt (Monitoring). Es ist ein wesentlicher Teil der Vision der digitalen Medizin, dass es möglich werden soll, diese umfassende Aufnahme von Daten mit medizinischem Wissen zu verbinden und so Entstehung, Verlauf und Behandlungsmöglichkeiten von Krankheiten aufzuklären und den Erhalt von Gesundheit zu fördern. Werden all diese Elemente in ein System integriert, sollen gesundheitsrelevante Prozesse simuliert werden können. Anhand von Simulationen soll es möglich werden, Krankheitsrisiken vorherzusagen. Nicht nur das, sondern es geht auch darum, alternative Szenarien durchzuspielen. Es soll möglich werden, Therapien auszuprobieren und präventives Verhalten auf seine Wirksamkeit hin zu testen. Mit solchen Technologien wird es 
möglich sein, die perfekten Maßnahmen zu ergreifen. So lautet das Versprechen der digitalen Medizin. Sie ist quantifizierbar, erlaubt Vorhersagen und informiert über Maßnahmen. Sie ist, wie es Hood und Flores formulieren: ,actionable“ (vgl. Hood und Flores 2012). So gesehen geht es hier nicht nur um ein Versprechen umfassenden Wissens, sondern auch um jenes einer umfassenden Kontrolle (Downing 2011, S. 70).

Die Funktion einer Vision besteht darin, eine Entwicklungsperspektive zu organisieren. Sie gibt eine Richtung für Forschungs- und Innovationsbemühungen vor (vgl. Pinch und Bijker 1984; Bijker 1995). Zwei Ideen sind für die strategische Ausrichtung der digitalen Medizin besonders wichtig: Es ist a) die Möglichkeit zur Prävention (durch prädiktive Simulation) und b) die Prüfung der Wirksamkeit eingesetzter Maßnahmen (Evidenzbasierung und Monitoring). Genau darauf bezogen, wird digitale Medizin als Option präsentiert, die perfekten technischen Mittel bereitzustellen.

\section{Prävention}

Der Gedanke der Prävention ist gleichermaßen einfach wie überzeugend. Präventive Medizin sucht nach geeigneten Maßnahmen, durch die erreicht werden kann, dass Krankheiten erst gar nicht auftreten (vgl. Leppin 2010). Eine solche Möglichkeit ist jedoch nicht voraussetzungslos. Präventive Maßnahmen können nur dann erfolgreich gesetzt werden, wenn die Ursache-Wirkung-Beziehungen von Krankheiten bekannt sind. Nur wenn man weiß, was Krankheiten auslöst und Gesundheit erhält oder wiederherstellt, ist es möglich, dementsprechend zielgerichtete Maßnahmen zu ergreifen. Aus diesem Grund hat das Wissen über die Kausalbeziehungen von Krankheiten eine zentrale Bedeutung. Dieses Wissen bereitzustellen, ist Aufgabe der Medizin, der sie durch intensive Forschungsleistungen nachzukommen bemüht ist. Spätestens seit dem Human-Genom-Projekt ist klar, dass das Leben und damit menschliche Gesundheit in hohem Maße komplex ist. Vor diesem Hintergrund gibt es begründete Zweifel daran, dass sich solche komplexen Systeme vorhersagen oder kontrollieren lassen. Dennoch halten etwa Tian et al. (2012) fest, dass medizinische Modellierungen prädiktiv sein MÜSSEN. Denn nur dann sind sie anwendbar und für die Behandlung von PatientInnen nützlich (Tian et al. 2012, S. 113).

Es ist eine machtvolle Vorstellung, über das Wissen zu verfügen, auf dessen Grundlage es gelingt, das Auftreten von Krankheiten zu vermeiden. Auf individueller Ebene ist ein Leben ohne Leiden und Einschränkungen, ja ein Leben ohne Ende ein Traum, den Menschen schon sehr lange träumen. Auf gesellschaftlicher Ebene wird die Möglichkeit zur Prävention sehr stark von ökonomischen Überlegungen befördert. Es ist billiger, präventive Maßnahmen zu setzen, als aufgetretene Krankheiten durch teure Therapien zu behandeln. Diabetes, Herzinfarkt und Schlaganfall sind dazu gut dokumentierte Beispiele. Zusammen nähren diese Träume und ökonomischen Erwägungen den „Willen zum Wissen“ (vgl. Foucault 1992 [1976]). Der Gewinn, den wir uns aus diesem Wissen über das präventive Verhindern von Krankheiten erträumen und erhoffen, ist zu groß, als dass man seiner Verlockung widerstehen könnte. 
Die Innovationsziele der digitalen Medizin korrespondieren mit diesem Willen zum Wissen. Mit digitalen Technologien soll es möglich werden, das erforderliche Wissen bereitstellen zu können. Medizinische Daten werden als Ressource gesehen und ihre Auswertung verspricht Erkenntnisse über wirkungsvolle Präventionsmaßnahmen. Dafür steht nicht zuletzt das Schlagwort Big Data. Gerade im medizinischen Kontext sind die Erwartungen hoch, durch die computergestützte Analyse großer Datenmengen neue Erkenntnisse zu gewinnen (vgl. Kolany-Raiser et al. 2018). In der medizinischen Literatur wird seit geraumer Zeit der Begriff „Precision Medicine“ verwendet, um die Potenziale digitaler Technologien in der Medizin hervorzuheben (vgl. Duffy 2016). „Precision medicine“ greift die Ziele der ,persolnalized medicine“ auf (vgl. dazu Prainsack 2017), wie sie schon im Kontext der Genomforschung vorangetrieben wurden, und erweitert diese durch den Einsatz digitaler Technologien. Obwohl der Fokus von „Precision Medicine“ derzeit vor allem auf der medizinischen Forschung liegt, bedeutet Wissen bereitzustellen, letztlich, es verfügbar zu machen. Digitale Technologien bringen das Wissen dorthin, wo es gebraucht wird. Dort, wo Handlungen gesetzt werden müssen, soll es zur richtigen Zeit am richtigen Ort sein, um seine präventive Wirksamkeit entfalten zu können.

Digitale Technologien haben zwei große Stärken: Sie sind sehr gut im Sammeln von Daten (Monitoring), und sie sind sehr gut im Durchführen von (algorithmisierbaren) Funktionsabläufen (Simulation) (vgl. Kelleher und Tierney 2018). Auf diesen beiden Ebenen setzen digitale Technologien an, um ihr Versprechen einzulösen, Wissen bereitzustellen und verfügbar zu machen, um so Krankheiten heilen, ja sogar verhindern zu können. Im Folgenden werden diese beiden Ebenen als Grundfunktionen digitaler Medizin charakterisiert: a) Monitoring und b) Simulation.

\section{Monitoring}

Mit den Technologien der digitalen Medizin wird es möglich, ein umfassendes Monitoring von medizinischen Daten zu realisieren. Dies beginnt mit dem Erfassen und Verarbeiten von Patientenakten und geht bis hin zur Dokumentation individuellen Verhaltes der Personen. Sensoren am Körper, im häuslichen Umfeld und in der Umwelt stellen Daten bereit, mit denen es möglich werden soll, ein Gesamtbild zu zeichnen. In Echtzeit können Ernährung, Bewegung, die Wirksamkeit von Medikamenten bis hin zur Einhaltung von Therapieplänen erhoben und protokolliert werden. Daten zur Steigerung von Fitness, Regeneration und Stress lassen sich so ebenfalls erheben, weiterverarbeiten und auswerten. Bousquet et al. sehen insbesondere im Kontext von chronischen Erkrankungen ein fortwährendes, individualisiertes und multidimensionales Screening als Teil einer präventiven Strategie der Gesundheitsoptimierung (vgl. Bousquet et al. 2011, S. 12). Doch geht es auch darum, grade jene Phasen des Lebens zu monitoren, in denen Menschen gesund sind (Hood 2013). Gesundheit und Krankheit werden unter diesem Vorzeichen als Prozesse erfasst, verstanden und ,individuell“ zu optimieren versucht (ibid.).

Dieses umfassende Monitoring in Echtzeit ist die erste wesentliche technische Möglichkeit, die mit der digitalen Medizin in Verbindung gebracht werden kann. Sind sie erhoben, ist es möglich, medizinische Daten weiterzugeben und mit anderen 
zu teilen. Dieser Kommunikationsaspekt ist bedeutsam. Hinzu kommt, dass die Weitergabe von Daten durch Internettechnologien automatisierbar wird (Internet of Things - IoT, siehe Chui et al. 2010; Car et al. 2019). Monitoring ist also nicht nur im Sinne der Datenerfassung mittels Sensoren umfassend, sondern auch über die informationstechnische Vernetzung alles umgreifend. Die Erfassung und Vernetzung ist allumfassend, zumindest ist sie in einem Ausmaß möglich, wie es ohne digitale Technologien undenkbar war.

\section{Simulation}

Digitale Technologien eröffnen die Möglichkeit zur Simulation. Computertechnologien stellen Mittel bereit, medizinische Vorgänge in silico nachzubilden. Das beginnt auf der molekularen Ebene und reicht bis zur Simulation der Gesundheitsfolgen von Lebensstil und Umweltbedingungen. Wo die Systemgrenzen einer Simulation gesetzt werden und welche Daten und Modelle integriert werden, ist Definitionssache. Der Umstand, dass Simulationen es ermöglichen, präventive und therapeutische Interventionen durchzuspielen, d.h. zu testen, wie sich einzelne Maßnahmen auf das simulierte System auswirken, wird hier als besonders bedeutsam erachtet.

Simuliert kann beispielsweise werden, wie die Moleküle eines Medikaments mit den genetischen Mutationen von Krebszellen chemisch interagieren. Simuliert werden kann auch, wie sich sportliche Betätigung auf den Stoffwechsel und etwa auch auf das Risiko von Herz-Kreislauf-Erkrankungen auswirkt. Den Möglichkeiten der Simulation sind keine Grenzen gesetzt, zumindest nicht in der Fantasie. Bedeutsam sind Simulationen deshalb, weil sie dazu herangezogen werden können, die Wirksamkeit von Interventionsmöglichkeiten sichtbar und überprüfbar zu machen.

In der medizinischen Literatur wird in diesem Zusammenhang stärker der Begriff ,Modellierung“ verwendet (englisch auch ,,predictive modelling“) (Duffy 2016, S. 495). Ziel der Quantifizierung ist es, das Leben in seiner Gesamtheit kalkulierbar zu machen (Vogt et al. 2016, S. 311). An der Schnittstelle zu den PatientInnen wird es durch einen Avatar, d.h. mit einem ,virtual physiological human“ möglich, genau dies technisch umzusetzen (vgl. Hunter et al. 2013). Computersimulationen sind demnach a) Mittel der Erkenntnis, b) Mittel der Erprobung und Prüfung, und c) sie sind mehr als nur ein Abbild der Wirklichkeit, aber sie brauchen eine umfassende Datenbasis als Ausgangspunkt. Ein System, welches all das kann, eine digitale Technologie, die menschliches Leben überwachen und simulieren kann, ist ein zentrales Entwicklungsziel digitaler Medizin (vgl. Diaz et al. 2013; Hunter et al. 2013).

\section{Gesunde Kranke}

Wird der Traum der Prävention Wirklichkeit, hat das weitreichende Implikationen. Pointiert formuliert, werden wir zu gesunden Kranken. Technologien, die dazu in der Lage sind, Krankheiten vorherzusagen, geben auch Aufschluss darüber, was getan werden kann, um das Auftreten von Krankheiten zu verhindern. Wird das Verspre- 
chen der digitalen Medizin eingelöst, lassen sich (alle) Krankheiten vorhersagen, und es lässt sich darüber hinaus angeben, was getan werden kann, um das Auftreten einer prognostizierten Krankheit abzuwenden. In letzter Konsequenz bedeutet das, dass zu einem Zeitpunkt mit der Therapie begonnen wird, wo die behandelte Krankheit noch gar nicht aufgetreten ist. Das meint der Begriff ,gesunde Kranke“. Personen, die bislang als gesund galten, werden nunmehr in einer neuen Weise objektiviert. Sie werden als behandlungspflichtig erachtet, bevor sie erkrankt sind. Prävention richtet sich nicht zuletzt gerade auf asymptomatische, gesunde Menschen (vgl. Flores et al. 2013, S. 567 sowie Bousquet et al. 2011; Diaz et al. 2013). Proponenten der digitalen Medizin machen den Nutzen dieser Möglichkeiten deutlich, indem sie etwa auf die Möglichkeit hinweisen, Männer prophylaktisch gegen Prostatakrebs zu behandeln, zumal $80 \%$ der über 50-Jährigen ein Risiko haben, daran zu erkranken. Hood argumentiert, dass es so möglich wäre, das Risiko jener potenziell Gefährdeten auf $2 \%$ zu reduzieren, begänne man all diese Männer mit $35 \mathrm{zu}$ behandeln (vgl. Hood 2008, S. 29). Den Traum der Prävention gibt es schon länger. Was mit den digitalen Technologien neu ist, ist die Möglichkeit der permanenten Kontrolle auch außerhalb der Klinik. Mit einem fortwährenden, allumfassenden Monitoring aller physiologischen, psychischen und sozialen Parameter kann im Augenblick ermittelt werden, wie sich der ermittelte Zustand auf die Gesundheit auswirkt und welche Maßnahmen getroffen werden können, um ein drohendes Gesundheitsrisiko umgehend abzuwenden.

Der Begriff ,gesunde Kranke“ tauchte im Kontext der Reflexion der genetischen Medizin auf (vgl. Nelkin 1995, S. 208, Lemke 2004, S. 77). Insbesondere durch die prädiktive genetische Diagnostik wurde bewusst, dass Krankheiten erkannt werden können, bevor sich Symptome zeigen. Genetische Tests eröffnen diese Option prädiktiver Diagnostik. Am deutlichsten wurde das am Beispiel von Chorea Huntington, einer sogenannten Late-onset-Erkrankung (i.e. eine Krankheit, die erst in fortgeschrittenem Lebensalter auftritt, vgl. Cox 1999). Die genetische Disposition kann bereits bei der Geburt oder sogar pränatal festgestellt werden. Die Krankheit Chorea Huntington kommt aber erst in der dritten oder vierten Lebensdekade zum Ausbruch. Solange dies nicht der Fall ist, spricht man von präsymptomatischen PatientInnen. Im Zuge der Erforschung des menschlichen Genoms wurden umfassende Möglichkeiten zur Vorhersage von Krankheitsrisiken und damit die Option zu präventivem Handeln in Aussicht gestellt.

Bereits in diesem Zusammenhang wurden Vorstellungen mobilisiert, auf Basis eines tiefen molekularen Verständnisses des Lebens die Möglichkeit zu gewinnen, dieses zu kontrollieren. Nikolas Rose sieht darin die Vision ,... that life can now be engineered" (Rose 2007, S. 4). Abgesehen davon, dass sich diese Hoffnungen als zu optimistisch herausgestellt haben, werden sie als Reduktionismus kritisiert. Vielleicht wenig überraschend begegnet man diesem Reduktionismusproblem nun damit, alles informationstechnisch zu erfassen und zu integrieren. So schlagen etwa Boissel et al. vor: „,The solution must include multilevel interactions in an integrative approach. Thus, systems medicine should go beyond the realm of the intercellular layer to integrate upper physiological layer, include all time and complextity leves components“ (Boissel et al. 2015, S. 4). Die digitale Medizin verspricht, diese Träume realisieren zu können. Sie ist immer zur Stelle, begleitet Menschen immer 
und überall, jede Kleinigkeit wird erfasst und ausgewertet. Sofort können Maßnahmen ergriffen und drohende Krankheitsrisiken abgewendet werden. Auf die Spitze getrieben bedeutet das, alle Menschen sind immer schon krank. Den Zustand der Gesundheit gibt es nicht mehr. Das Risiko zu erkranken besteht immer. Was man tun kann (und auch tun muss), ist, dieses Risiko zu minimieren. Diese Tendenz hat auch damit zu tun, dass sich Medizin unter dem Vorzeichen der Biomedikalisierung (vgl. Clarke et al. 2003) - dem allumfassenden Zugriff auf das Leben insgesamt, der bereits mit der genetischen Medizin einsetzte - in einem allumfassenden Sinn für Gesundheit zuständig sieht und nicht mehr bloß für Krankheit als deren Gegenteil (vgl. Downing 2011, S. 2).

\section{Neue Verhaltensimperative}

$\mathrm{Ob}$ und wodurch aus der Möglichkeit, etwas zu tun, eine Verpflichtung wird, ist eine relevante Frage. Auch sie wurde bereits im Kontext der genetischen Medizin eingehend diskutiert. Thomas Lemke und andere sprechen vom Entstehen neuer Verhaltensimperative und meinen damit die Verpflichtung zu risikominimierendem Gesundheitsverhalten (vgl. Lemke 2004, 2000; siehe auch Clarke et al. 2003, S. 21). Diese Imperative werden durch den Umstand verstärkt, dass wirksame Handlungsoptionen zur Verfügung stehen. Je mehr es möglich wird, durch eigenes Verhalten die eigene Gesundheit positiv zu beeinflussen, desto stärker der Imperativ, auch etwas tun zu müssen.

Auffallend ist dabei, dass der Imperativ in Verbindung mit einem Eigennutzen vorgetragen wird: Du wirst es sein, der/die profitiert. Für dich selbst musst du es tun! Auf diese Weise käme es - so lautet das Argument - zu einer Verlagerung der Verantwortung. Das Individuum sei selbst für seine Gesundheit verantwortlich (vgl. Lemke 2004, S. 17). Trotz dieser Individualisierung von Verantwortung kann zugleich von einem gesellschaftlichen Druck gesprochen werden, der diese Individualisierung von Verantwortung befördert. Weil auftretende Erkrankungen Kosten für die Allgemeinheit verursacht, resultiert daraus die Verpflichtung, auf die eigene Gesundheit zu achten. Diese Verpflichtung entsteht aus der Sozialisierung der Kosten des öffentlichen Gesundheitssystems. Die Unterlassung der Möglichkeit, Krankheitsrisiken zu vermeiden und förderliche Maßnahmen zu ergreifen, wird vor diesem Hintergrund moralisch fragwürdig. Vogt und andere weisen darauf hin, dass solche Imperative des Partizipieren-Müssens Teil der personalisierten Medizin sind (Vogt et al. 2016, S. 318). Die Autoren sehen eine zunehmende Verpflichtung, medizinische Daten zu teilen und an WissenschaftlerInnen und ÄrztInnen weiterzuleiten. In der medizinischen Fachliteratur wird das etwa von Bousquet und Kollegen explizit so gefordert, die es für die soziale Verantwortung von PatientInnen halten, dass diese ihre Daten verfügbar machen (vgl. Bousquet et al. 2011, S. 3). Dementsprechend halten Fiske, Prainsack und Buyx resümierend fest: „There are increasing expectations that individuals make informed decisions as responsible managers of their health, and now also as owners (morally or legally) of their data" (Fiske et al. 2019, S. 3). Vegter sieht diese spezifische Form von Verantwortung nicht zuletzt mit 
einer intensivierten Praxis des ,self-monitoring“ und „self-management“ verbunden (Vegter 2018, S. 445).

Die Deutung dieser neuen Imperative als Teil neo-liberaler Regierungsweisen wurde insbesondere von Thomas Lemke ausgearbeitet (Lemke 2007, 2004b, 2003, 2000). In der englischsprachigen Literatur sind hier noch Nicolas Rose und Carlos Novas zu nennen (vgl. Miller und Rose 2008; Novas und Rose 2000; Rose 1999, 2007; Rose und Novas 2005). Regiere dich selbst, du wirst profitieren! Wesentlich ist für Lemke in diesem Zusammenhang, dass „Krankheiten als Risiken konzipiert und Risiken als Krankheiten“" aufgefasst werden (Lemke 2004, S. 93). Lemke führt dazu weiter aus:

Die Identifikation von Krankheiten ist kein deskriptiv-wissenschaftlicher Vorgang, sondern impliziert immer ein normatives Urteil über die Veränderungsbedürftigkeit von Zuständen. Das genetische Wissen allein kann keinen Beitrag zu der Frage leisten, ob ein Zustand behandelt werden soll oder nicht. Es ist vielmehr mit expliziten Gründen zu urteilen, welche „Abweichungen“ als pathologisch charakterisiert und vermieden bzw. behandelt werden sollen. (Lemke 2004, S. 93).

Der Rekurs auf die genetische Medizin ist hilfreich. Aus einer elaborierten Reflexion lassen sich zahlreiche Thesen ableiten, die, bezogen auf die digitale Medizin, zu prüfen sich lohnt. Der Analogieschluss wäre jedoch voreilig. Selbst wenn zwischen den genannten Bereichen Ähnlichkeiten konstatiert werden können, so ist es dennoch wichtig, digitale Medizin eigenständig zu betrachten und bekannte Thesen erneut und kontextspezifisch zu hinterfragen. Im Folgenden möchte ich nun erläutern, in welcher Weise digitale Medizin über das hinausgeht, was bereits im Kontext der genetischen Medizin diskutiert wurde.

\section{Interventionsmodus}

Medizinisches Wissen soll den Menschen zugutekommen, es soll helfen, Krankheiten zu heilen, ja, deren Auftreten überhaupt verhindern. Medizinische Forschung strebt nach Erkenntnissen, die nicht bloß Krankheitsursachen ergründen und den biologischen Funktionen des menschlichen Organismus auf den Grund gehen, sondern letztlich geht es darum, Wissen hervorzubringen, auf dessen Grundlage ableitbar ist, was konkret getan werden kann. Mit anderen Worten, medizinisches Wissen muss „actionable“ sein. Diese Handlungsmöglichkeiten (,,actionable possibilities“) zählen zu den zentralen Elementen moderner Präzisionsmedizin (vgl. Hood et al. 2015, S. 1). Gesundheitsförderndes Verhalten liegt jedoch nicht zuletzt in der Verantwortung der Einzelnen, wie es die zuvor erörterten Imperative nahelegen. Im Folgenden möchte ich zeigen, wie sich mit digitalen (Interface-)Technologien der Modus ändert, durch den medizinisches Wissen auf individueller Ebene handlungswirksam wird.

Zieht man den Vergleich zur genetischen Medizin, so wird der Unterschied besonders deutlich. In der genetischen Medizin war und ist die ,humangenetische Beratung“ der zentrale Interventionsmodus (vgl. Reif und Baitsch 1986; Buselmaier 
und Tariverdian 1991; Hartog 1996). In einem Gespräch werden die Individuen (sie werden im Jargon als „Ratsuchende“ bezeichnet) über die medizinischen Hintergründe ihrer genetischen Konstitution in Kenntnis gesetzt. Das Beratungsgespräch beinhaltet neben der Vermittlung von medizinischem Wissen auch das Aufzeigen von Handlungsmöglichkeiten. Es ist zentral, dass die vorgestellten Optionen als gleichmögliche Alternativen nebeneinandergestellt werden. Die Entscheidung, welche Handlungsoption gewählt wird, soll alleine bei den Betroffenen liegen. Das ist das Prinzip und ethische Fundament der ,nichtdirektiven Beratung“ in der genetischen Medizin.

Wenn die wesentliche Integrationsleistung von medizinischem Wissen und gesundheitsbezogenem Handeln durch das betroffene Individuum geleistet werden muss, dann operieren diese Modelle über die Figur des autonomen Subjekts (vgl. Berger 2006, 2010). Dieses autonome Subjekt ist vernunftbegabt und in der Lage, das eigene Verhalten selbst zu steuern. Die Urteilsfähigkeit (des autonomen Subjekts) ist Voraussetzung dafür, dass die ärztliche Empfehlung vom Individuum integriert und umgesetzt wird. Aufforderungen wie beispielsweise „Stellen Sie Ihre Ernährung um und bewegen Sie sich mehr!" werden nur dann handlungswirksam, wenn sie vom betroffenen Individuum selbst integriert und in die Tat umgesetzt werden. Medizinisches Wissen, Beobachtung des eigenen Verhaltens und Umsetzung in konkrete Handlungen, all das ist vom - im Kant'schen Sinne - vernünftigen und urteilsfähigen Subjekt umzusetzen. Dieses Subjekt wird darüber hinaus in dem Sinne als autonom gedacht, als es in seinen Entscheidungen und Handlungen frei ist, das eine oder auch das andere zu tun.

Mit den Möglichkeiten der digitalen Medizin ändert sich dieses Paradigma radikal. Am deutlichsten wird das, wenn man an einen Avatar denkt. Die Grundidee eines digitalen Zwillings besteht darin, dass durch Informationstechnologien umfassende medizinische Daten über ein konkretes Individuum gesammelt und über eine Computersoftware mit Verhaltensdaten und medizinischem Wissen zusammengeführt werden. Digitale Technologien können Daten sammeln, auswerten und über die Integration (Operationalisierung) von medizinischem Wissen Handlungsempfehlungen artikulieren. Auf diese Weise wird die steuernde Instanz externalisiert. Das vernünftige und urteilsfähige Subjekt wird obsolet. Es muss sich weder selbst beobachten noch verstehen, wie sich das eigene Verhalten auswirkt, noch muss es Schlüsse ziehen und entscheiden, welche Handlungen (im Sinne der eigenen Gesundheitsförderung) zu setzen sind. Diese Leistungen werden an digitale Technologien übertragen. Konsequenterweise sind Individuen, die sie verwenden, keine autonomen Subjekte. Sie sind es deshalb nicht, weil sie ihre Existenz mit der zum Einsatz kommenden Technologie teilen.

\section{Cyborgs}

Das Zusammenspiel zwischen Mensch und Technologie im Kontext digitaler Medizin verdient besondere Beachtung. Hier ist es vielleicht deutlicher als sonst, dass durch die Verbindung von Menschen und Technologien etwas Neues entsteht, das nur dann verstanden werden kann, wenn man es gemeinsam und miteinander verbunden 
betrachtet. ${ }^{4}$ Menschen und digitale Technologien - seien es Avatare, Fitnessuhren (Wearables) oder auch nur auf dem Smartphone installierte Apps - sind demnach nicht als getrennte Entitäten, sondern am besten als zusammengehörige sozio-technische Einheit zu begreifen (vgl. Lupton 2013, S. 39; Latour 2000). Die Implikationen sind weitreichend.

Unter den Voraussetzungen der digitalen Medizin macht eine Subjekt-ObjektDichotomie keinen Sinn mehr. ${ }^{5}$ Die dem Subjekt zugeordneten Eigenschaften werden durch digitale Technologien externalisiert. Wahrnehmungen, Sinneseindrücke werden von Sensoren übernommen und in Daten übersetzt, Entscheidungen werden durch Computer-Algorithmen vorstrukturiert oder überhaupt vorweggenommen. Unter diesen Voraussetzungen gibt es kein autonomes Subjekt mehr. Zentrale Begriffe der Medizinethik, wie Selbstbestimmung und „Informed Consent“, machen keinen Sinn mehr. Sie alle setzen ein autonomes Subjekt voraus, das sich bereits in vielfacher Weise mit digitalen Technologien vernetzt hat, Daten teilt, seinen Körper über Zahlen auf einem Display wahrnimmt und Handlungsanweisungen folgt, die es selbst nicht mehr kognitiv prozessiert hat. In einem tiefgreifenden Sinn machen die Worte „Entscheidung“ und „Verantwortung“ für einen Cyborg keinen Sinn mehr - nicht, wenn eine Entscheidung so verstanden wird, dass sie von autonomen Subjekten getroffen und verantwortet wird. Die Vorstellung von dem, was wir Subjekt nennen, ändert sich radikal. Selbst jene, die Haraways Cyborgs und Latours Aktanten mit Skepsis betrachten, werden einsehen, wie fundamental sich eine Existenz ändert, wenn sie in einer Weise vernetzt ist, wie es mit digitalen Technologien möglich wird (vgl. Haraway und Hammer 1995; Latour 1994, 2000).

\footnotetext{
${ }^{4}$ Donna Haraway hat dafür den Begriff „Cyborg“ vorgeschlagen und Bruno Latour das Wort „Aktant“ (vgl. Haraway und Hammer 1995; Latour 1994, 2000).

${ }^{5}$ Die Trennung von Subjekt und Objekt geht auf René Descartes zurück und wurde zur wesentlichen philosophischen Grundlage der modernen Naturwissenschaften (Foucault 1994 [1987], S. 290-291; Braidotti 2014, S. 61). Insbesondere für eine naturwissenschaftlich orientierte Medizin, in welcher der menschliche Körper zum Objekt der Erkenntnis wird, ist diese philosophische Grundlage elementar (vgl. Foucault 1993 [1963]). Diese Dichotomie von Subjekt und Objekt setzt sich in der Aufklärung fort, wo sie einerseits in Kants ,transzendentalem“ Subjekt weitergedacht und andererseits zur philosophischen Voraussetzung für das ,autonome Subjekt“ wird, das für sich selbst Entscheidungen trifft. Wie oben bereits argumentiert, gerät die Figur eines als ,autonom“ gedachten Subjekts zunehmend in Kritik. Die Arbeiten von Michel Foucault stehen hier am Anfang. Später haben Bruno Latour und Donna Haraway ebenfalls dafür plädiert, die als gegeben erachtete Subjekt-Objekt-Trennung zu überdenken und stattdessen zu einem Verständnis zu kommen, welches es ermöglicht, das Subjekt als hybrid und mit der Objektwelt verbunden zu denken (Haraway und Hammer 1995; Latour 1994, 2000). Was bei Latour und Haraway mitunter abstrakt und theoretisch klingt, ist mit den modernen, digitalen Technologien zur alltagsweltlichen Erfahrung geworden. In unserer komplexen, interdependenten Welt, in der wir zu jeder Zeit technologisch mit anderen Menschen und Maschinen verbunden sind und permanent Datenspuren hinterlassen, wird es zunehmend zur Illusion, anzunehmen, dass wir ,autonom“ agieren, indem wir per Mausklick den „,Allgemeinen Geschäftsbedingungen“ von Computerprogrammen zustimmen oder unseren „Informed Consent“ per Unterschrift erklären. Gerade in der medizinethischen Reflexion werden die Grenzen eines ,autonomen“, „Informed Consent"-gebenden Subjekts seit geraumer Zeit kritisiert (vgl. Maier 2006; Sariola und Simpson 2011). Die hier vorgeschlagene Perspektive erfordert es, gewohnte und tief in die westliche Philosophie eingeschriebene Grundannahmen und Denkfiguren infrage zu stellen. Das mag zu Irritationen führen, eröffnet aber neue - und aus meiner Sicht überaus fruchtbare - Zugänge für die kritische Reflexion digitaler Interface-Technologien.
} 


\section{Gesundheitsbegriff}

Medizin hat nicht bloß die physiologische Behandlung von Krankheiten im Blick, sondern auch psychologische Elemente, und orientiert sich an der gesamten Lebensweise der Menschen. Die Grenzen zu Wohlbefinden und Wellness sind längst überschritten und nunmehr klar im Fokus medizinischen Handelns (vgl. Hood et al. 2015; Flores et al. 2013). Moderne Medizin erhebt zwar den Anspruch auf eine allumfassende Betrachtung der Individuen, doch operiert dieser „Holismus“ quantitativ. Auch wenn Gesundheit nunmehr die psychologische Ebene miteinschließt, so ist Wohlbefinden nichts, was empfunden, sondern vielmehr gemessen wird. Digitale Medizin macht Gesundheit zu einer Steuerungsgröße; zu etwas, das man messen und auf eine Zielvorgabe beziehen kann. ${ }^{6}$ Ich meine damit nicht, dass Menschen unter Verwendung digitaler Technologien keine, weniger oder auch nur andere Gefühle haben (vgl. Duttweiler et al. 2016). Relevant ist vielmehr, wie ein Normwert entsteht, der zum Abgleichen mit dem eigenen Gesundheitszustand herangezogen wird (vgl. Tutton 2014 sowie Foucault 1992 [1976], 1991 [1975], 1993 [1963]).

Die Entstehung von Normwerten wird deutlich, wenn man an die Festlegung von idealen Blutdruckwerten denkt. Wie hoch sind normale Werte? Ab wann ist ein bestimmter Blutdruckwert ungesund oder sogar krank? Die zur Beantwortung solcher Fragen erforderliche Norm kann durch die Ermittlung des Durchschnittswertes festgelegt werden. Normal ist demzufolge das statistische Mittelmaß. Doch ist dieser Wert auch gesund? Man kann sich Algorithmen vorstellen, durch welche ermittelt wird, welche Blutdruckwerte mit der höchsten Lebenserwartung korrelieren. Wird eine solche Ermittlung technisch möglich, kann der entsprechende Wert nun als Zielwert festgelegt werden. Wie auch immer solche Normwerte festgelegt werden, eines ist klar: Sie werden sicherlich nicht über das individuelle Wohlbefinden ermittelt. Was die WHO vor vielen Jahrzehnten als ,physisches, psychisches und soziales Wohlbefinden" formuliert hat, erscheint nunmehr in einem neuen Licht (vgl. WHO 1948). Der Punkt ist, dass sich der Gesundheitsbegriff mit den Möglichkeiten digitaler Technologien zunehmend in operationalisierbare Steuerungsgrößen verwandelt. Die Quantifizierbarkeit ist zentral, denn sie ist Voraussetzung dafür, dass ein Abgleich stattfinden kann, aus dem sich Handlungsanweisungen ableiten lassen.

Es ist völlig konsistent mit dieser Form der Rationalität (sie wird mitunter auch „computational thinking“ genannt), einen Zielwert durch einen anderen zu ersetzen (vgl. Kelleher und Tierney 2018). Die Norm, mit der die Werte eines Individuums abgeglichen werden, kann aus ihrer Korrelation mit maximaler Lebenserwartung ermittelt werden. Doch ist es genauso möglich, einen Algorithmus zu definieren, der Lebenserwartung mit Leistungsfähigkeit ersetzt. Der Gedanke der Leistungsoptimierung ist Medizinern durchaus nicht fremd. So schreiben etwa Boissel und andere: „The optimization of wellness is a key to maximizing human potential for each individual - improving physiological as well as psychological performances“ (Boissel et al. 2015, S. 10). Aus dieser Perspektive wird „Gesundheit“ als Kapazität definiert, die Menschen leistungsfähiger macht, sie schneller laufen, länger durchhal-

\footnotetext{
6 David Armstrong argumentiert ähnlich hinsichtlich molekulargenetischer Tumordiagnostik (vgl. Armstrong 2019).
} 
ten, mehr arbeiten, schneller denken und weniger Fehler machen lässt. Es gibt keine technische Barriere, Normvorgaben und Vergleichswerte, die nicht mit solchen Algorithmen zu ermitteln sind. Die Grenzen zwischen kurativer und wunscherfüllender Medizin verschwimmen auch aus anderen Gründen zunehmend. Durch den Einsatz digitaler Technologien kann diese Transgression weiter vorangetrieben werden.

Normvorgaben sind in dieser Hinsicht „,neutral“, sie können auf alle denkbaren Parameter hin ermittelt und optimiert werden. In der digitalen Medizin wird Gesundheit formal bestimmt, über den Modus ihrer Ermittlung, nicht jedoch durch inhaltliche Kriterien. Digitale Gesundheit ist, was sich operationalisieren lässt, was sich quantifizieren und mit Normwerten abgleichen lässt. Es ist der Versuch, Gesundheit vollständig zu quantifizieren (vgl. Hood 2013; Flores et al. 2013). All das, was man so nicht erfassen kann oder so nicht erfasst wird, hat nichts mehr mit Gesundheit oder Krankheit zu tun. Genau das sagt Lemke über genetische Tests: Sie stellen Abweichungen fest, doch sie können diese nicht bewerten. Das zu tun, erfordert einen gesellschaftlichen Diskurs.

\section{Optimierung}

Definiert man Gesundheit im oben vorgestellten Sinn und operationalisiert man sie aus dieser Perspektive, so wird Gesundheit zu etwas, das man optimieren kann (vgl. Rose 2007; Lupton 2013; Duttweiler et al. 2016). Gesundheitsvorsorge wird zur Optimierungspraxis. Wer beginnt, Ist- und Sollzustände zu vergleichen, wird es naheliegend finden, dieses Kalkül um Input- und Output-Vergleiche zu erweitern und nach Optimierung zu streben. Was unter Minimierung der aufgewendeten Mittel als maximal gesundheitsfördernde und risikominimierende Wirkung ermittelt werden kann, gehört zu diesem Kalkül. Damit ist angesprochen, was Ulrich Bröckling und andere die „Ökonomisierung des Sozialen“ nennen (vgl. Bröckling 2007; Bröckling et al. 2000; Bröckling 2017). Ökonomisierung ist hier im doppelten Sinn gemeint. Einerseits geht es darum, was sich monetär rechnet, was billiger ist und letztlich weniger Kosten für die steuerzahlende Allgemeinheit bedeutet. Ökonomisierung von Gesundheit ist auch in diesem Sinne angesprochen, doch es geht darüber hinaus: Kosten-Nutzen-Betrachtungen sind Teil eines ökonomischen Kalküls auch dann, wenn sie nicht in Euro oder Dollar gemessen werden. Die „Währung“ kann auch Lebenszeit oder -qualität sein. Wenn man es für zielführend hält, kann man sogar Wohlbefinden einsetzen. Es geht um die Rationalität. Wer sich fragt „Wie viel Aufwand ist mir der Zuwachs an Wohlbefinden wert? Was bin ich bereit dafür zu tun?“, der ist schon mitten im Versuch, das eigene Leben zu optimieren. Gesundheit wird zur Humanressource (vgl. Lemke 2000).

Diese ökonomische Optimierungslogik der digitalen Medizin ist nicht äußerlich - nichts, was man weglassen oder anders machen könnte. Vor dem Hintergrund des Dargestellten lässt sich argumentieren, dass die Optimierungslogik ein inhärenter Bestandteil der digitalen Medizin ist. Würde das bedeuten, dass digitale Medizin ohne diese Optimierungslogik, ohne eine Ökonomisierung des Sozialen, gar nicht möglich ist? Vielleicht. Empirische Studien werden zeigen, in welcher Form sie tatsächlich umgesetzt und angewandt wird. 


\section{Schlussfolgerungen}

Der Einsatz digitaler Technologien in der Medizin gehört zweifellos zu den großen Hoffnungsträgern der Gegenwart. Potenziale werden im gesamten Bereich gesehen, begonnen bei der Verwaltung medizinischer Daten, über den Einsatz in der Forschung bis hin zu Gesundheitsvorsorge, Behandlung und Pflege. Derzeit befinden sich diese Technologien weitgehend noch im Entwicklungsstadium. Zwar finden sich bereits einzelne Produkte auf dem Markt, doch gemessen an den enormen Potenzialen, die diesen Technologien zugesprochen werden, ist die digitale Medizin derzeit in erster Linie ein Zukunftsversprechen. In diesem Text ging es darum, nach den Implikationen dieser Entwicklungsziele zu fragen. Ein Vision-Assessment erlaubt jedoch keine Prognosen soziotechnischer Entwicklungen. Stattdessen zielte diese Methode der Technikfolgenabschätzung auf die Deliberation der Wünschbarkeit von Zukunftsperspektiven. Vor diesem Hintergrund geht es darum, Fragen nach der Wünschbarkeit der vielfältigen Anwendungsmöglichkeiten digitaler Technologien im medizinischen Kontext zu diskutieren.

Digitale Medizin verspricht neue und bessere Methoden zu Diagnose und Therapie von Krankheiten und insbesondere die Möglichkeit zur Prävention. In Aussicht gestellt werden darüber hinaus die Steigerung des Wohlbefindens (Wellness) und der Leistungsfähigkeit (Enhancement). Eingelöst sollen diese Versprechen werden, wenn die dazu erforderlichen Technologien Marktreife erlangt haben und so lückenloses Monitoring und umfassende Simulationen möglich werden. Die vollständige und permanente Erfassung sämtlicher physischer, psychischer und sozialer Aspekte des Lebens erfasst alle Menschen als ,,potenziell krank“. In der sozialwissenschaftlichen Literatur wurden Zweifel an der Wünschbarkeit dieser Objektivierung mit dem Begriff ,gesunde Kranke“ artikuliert. Auch haben bereits die Erfahrungen mit prädiktivem Testen gezeigt, dass es durchaus nicht zwingend zu einer besseren Lebensqualität beiträgt, frühzeitig von genetisch angelegten Krankheiten zu erfahren. Mittlerweile herrscht vor allem dort deutlich mehr Zurückhaltung, wo Krankheiten zwar vorhergesagt, aber nicht geheilt werden können. Das prädiktive Potenzial umfassender Simulationen liegt auf einer vergleichbaren Ebene. Aus diesem Grund sind die durch die digitale Medizin in Aussicht gestellten Handlungsmöglichkeiten besonders wichtig.

Brisant ist die Frage, nach dem Interventionsmodus digitaler Technologien an der Schnittstelle zum individuellen (Gesundheits-)Verhalten. Das Ideal der informierten Entscheidung, die von jedem Individuum autonom getroffen werden kann, lässt sich schwer als Element eines Systems denken, das Handlungsentscheidungen unentwegt Hinweise zur Optimierung des Gesundheitsverhaltens gibt. Der versprochene Nutzen eines Avatars besteht ja gerade darin, klare Hinweise darauf zu geben, was getan werden kann, um Gesundheit, Wohlbefinden und Leistungsfähigkeit zu fördern. Autonom sind solche Entscheidungen nicht, vielmehr werden sie technologisch externalisiert und algorithmisiert. Digitale Technologien prozessieren Gesundheit als quantifizierbare Zielvorgabe. Ist das menschliche Leben erst einmal in Zahlen übersetzt, lässt es sich auch durchoptimieren. Gesundheitsverhalten wird so zur Optimierungspraxis. Anders ausgedrückt ermöglicht digitale Medizin eine Intensivierung der Ökonomisierung des Sozialen. Mit der technischen Umsetzung in Form eines di- 
gitalen Zwillings (Avatars) ist zur Verfügung gestelltes medizinisches Wissen nicht mehr äußerlich, sondern Bestandteil des digitalen ,virtual physiological human“. Dieser neuen Existenzform werden traditionelle Subjekt-Objekt-Dichotomien nicht gerecht. Ein Subjektverständnis ist erforderlich, dem es gelingt, der Hybridisierung von Mensch und digitalem Zwilling gerecht zu werden. So schlägt der französische Philosoph Bruno Latour vor, diese Dichotomie zu überwinden und das Verhältnis von Subjekt und Objekt neu zu denken. Dies ist gerade dann relevant, wenn das Zusammenspiel von digitalen Technologien und menschlichen Körpern verstanden werden soll. Menschen, die Sensoren am Körper tragen, sich über digitale Technologien mit anderen vernetzen, ihre Daten auswerten lassen und die Ableitung von Handlungen auf diese Weise externalisieren, deren Wirkungen sie wiederum mit digitalen Technologien prüfen, sind zu Mischwesen geworden, die sich nicht mehr trennscharf in menschliche Subjekte und technische Objekte aufgliedern lassen. Doch wenn wir durch und mit digitalen Technologien zu Aktanten - oder wie es Donna Haraway formuliert: zu Cyborgs - geworden sind, dann muss überdacht werden, was wir meinen, wenn wir von Verantwortung sprechen. Selbst das Wort Entscheidung verliert seine konventionelle Bedeutung, wenn man nicht mehr weiß, wer es ist, der oder die hier entscheidet. Ein autonomes Subjekt ist es nicht mehr.

Danksagung Das Projekt wurde von Prof. Dr. Kurt Zatloukal von der Medizinischen Universität Graz geleitet. Zentralen Anteil an den Projektarbeiten hatten Dr. Heimo Müller, Dr. Martina Lang, Robert Reihs, BSc., Simone Findling, Lukas Pessl, MA und Dr. Bernhard Wieser, beteiligte Institutionen waren neben der Medizinischen Universität Graz, das IFZ - Interdisziplinäres Forschungszentrum für Technik, Arbeit und Kultur und die Technische Universität Graz. Ich danke Hubert Eichmann für seine unterstützende Begleitung dieser Publikation.

Open Access Dieser Artikel wird unter der Creative Commons Namensnennung 4.0 International Lizenz (http://creativecommons.org/licenses/by/4.0/deed.de) veröffentlicht, welche die Nutzung, Vervielfältigung, Bearbeitung, Verbreitung und Wiedergabe in jeglichem Medium und Format erlaubt, sofern Sie den/die ursprünglichen Autor(en) und die Quelle ordnungsgemäß nennen, einen Link zur Creative Commons Lizenz beifügen und angeben, ob Änderungen vorgenommen wurden.

Förderung Dieser Beitrag wurde auf Basis des Projekts „Modelling Health“ (PN 8008) geschrieben. Das Projekt wurde im Rahmen des Zukunftsfonds des Landes Steiermark in der Ausschreibung zum Thema „Human Technology Interface: Healthy Ageing - Assisted Living (HTI:HA-AL) gefördert.

Funding Open access funding provided by Graz University of Technology.

\section{Literatur}

Antony, Paul M.A., Rudi Balling, und Nikos Vlassis. 2012. From systems biology to systems biomedicine. Current Opinion in Biotechnology 23(4):604-608.

Armstrong, David. 2019. Diagnosis: From classification to prediction. Social Science \& Medicine https:// doi.org/10.1016/j.socscimed.2019.112444.

Auffray, Charles, und Leroy Hood. 2012. Editorial: systems biology and personalized medicine-the future is now. Biotechnology Journal 7(8):938-939.

Berger, Wilhelm. 2006. Towards an ethics of distributed responsibility. In Prenatal testing: individual decision or distributed action?, Hrsg. Bernhard Wieser, Sandra Karner, und Wilhelm Berger, 13-26. München, Wien: Profil.

Berger, Wilhelm. 2010. Ethik als Machtprozess. In Ethik organisieren. Handbuch der Organisationsethik, Hrsg. Thomas Krobath, Andreas Heller, 604-616. Freiburg: Lambertus. 
Bijker, Wiebe E. 1995. Of bicycles, bakelites, and bulbs. Toward a theory of sociotechnical change. Cambridge: MIT Press.

Bijker, Wiebe E. 2010. How is technology made?-That is the question! Social Studies of Science 34(1):63-76.

Bijker, Wiebe E., und John Law (Hrsg.). 1992. Shaping technology/building society: Studies in sociotechnical change. Cambridge: MIT Press.

Boissel, J.-P., C. Auffray, D. Noble, L. Hood, und F.-H. Boissel. 2015. Bridging systems medicine and patient needs. CPT: Pharmacometrics \& Systems Pharmacology https://doi.org/10.1002/psp4.26.

Bousquet, Jean, Josep M. Anto, Peter J. Sterk, et al. 2011. Systems medicine and integrated care to combat chronic noncommunicable diseases. Genome Medicine 3(7):43.

Braidotti, Rosi. 2014. Posthumanismus. Leben jenseits des Menschen. Frankfurt, New York: Campus.

Bröckling, Ulrich. 2007. Das unternehmerische Selbst. Soziologie einer Subjektivierungsform. Frankfurt a. Main: Suhrkamp.

Bröckling, Ulrich. 2017. Gute Hirten führen sanft: Über Menschenregierungskünste. Berlin: Suhrkamp.

Bröckling, Ulrich, Susanne Krasmann, und Thomas Lemke (Hrsg.). 2000. Gouvernementalität der Gegenwart. Studien zur Ökonomisierung des Sozialen. Frankfurt a. Main: Suhrkamp.

Buselmaier, Werner, und Gholamali Tariverdian. 1991. Humangenetik: Begleittext zum Gegenstandskatalog. Heidelberg, Berlin, New York: Springer.

Car, Josip, Aziz Sheikh, Paul Wicks, und Marc S. Williams. 2019. Beyond the hype of big data and artificial intelligence: building foundations for knowledge and wisdom. BMC Medicine 17(1):143.

Chui, Michael, Markus Löffler, und Roger Roberts. 2010. The internet of things. McKinsey Quarterly March., 1-6.

Clarke, Adele E., Laura Mamo, Jennifer R. Fishman, Janet K. Shim, und Jennifer R. Fosket. 2003. Biomedicalization: technoscientific transformations of health, illness, and U.S. biomedicine. American Sociological Review 68(2):161.

Collingridge, David. 1980. The social control of technology. London: Pinter.

Cox, Susan M. 1999. “It's not a secret but ...”'Predictive testing and patterns of communication about genetic information in families at risk for Huntington disease. Vancouver: Doctoral Dissertation, University of British Columbia

Diaz, Vanessa, Marco Viceconti, Veli Stroetmann, und Dipak Kalra. 2013. Roadmap for the digital patient. Bruexelles: European Commission.

Downing, Raymond. 2011. Biohealth: beyond medicalization: imposing health. Eugene: Pickwick Publications.

Duffy, David J. 2016. Problems, challenges and promises: perspectives on precision medicine. Briefings in Bioinformatics 17(3):494-504.

Duttweiler, Stefanie, Robert Gugutzer, Jan-Hendrik Passoth, und Jörg Strübing (Hrsg.). 2016. Leben nach Zahlen: Self-Tracking als Optimierungsprojekt? Bielefeld: transcript.

Fiske, Amelia, Barbara Prainsack, und Alena Buyx. 2019. Data work: meaning-making in the era of datarich medicine. Journal of Medical Internet Research https://doi.org/10.2196/11672.

Flores, Mauricio, Gustavo Glusman, Kristin Brogaard, Nathan D. Price, und Leroy Hood. 2013. P4 medicine: how systems medicine will transform the healthcare sector and society. Personalized medicine 10(6):565-576.

Foucault, Michel. 1991. Überwachen und Strafen. Die Geburt des Gefängnisses. Frankfurt a. Main: Suhrkamp.

Foucault, Michel. 1992. Der Wille zum Wissen. Sexualität und Wahrheit, Bd. 1. Frankfurt a. Main: Suhrkamp.

Foucault, Michel. 1993. Die Geburt der Kliniken. Eine Archäologie des ärztlichen Blicks. Frankfurt a. Main: Suhrkamp.

Foucault, Michel. 1994. Zur Genealogie der Ethik. Ein Überblick über laufende Arbeiten. Interview von Hubert L. Dreyfus und Paul Rabinow. In Michel Foucault. Jenseits von Strukturalismus und Hermeneutik, Bd. 2, Hrsg. Hubert L. Dreyfus, Paul Rabinow, 264-292. Frankfurt a. Main: Athenäum.

Grasse, Christian, und Ariane Greiner. 2013. Mein digitales Ich. „wie die Vermessung des Selbst unser Leben verändert und was wir darüber wissen müssen“, 1. Aufl., Berlin: Metrolit.

Grunwald, Armin. 2010. Technikfolgenabschätzung - Eine Einführung. Berlin: edition Sigma.

Habermas, Jürgen. 2001. Die Zukunft der menschlichen Natur. Auf dem Weg zu einer liberalen Eugenik? Frankfurt a. Main: Suhrkamp.

Haraway, Donna J., und Carmen Hammer (Hrsg.). 1995. Die Neuerfindung der Natur: Primaten, Cyborgs und Frauen. Frankfurt a. Main: Campus. 
Hartog, Jennifer. 1996. Das genetische Beratungsgespräch: Institutionalisierte Kommunikation zwischen Experten und Nicht-Experten. Tübingen: Narr.

Hood, Lee. 2008. A personal journey of discovery: developing technology and changing biology. Annual Review of Analytical Chemistry 1(1):1-43.

Hood, Leroy. 2013. Systems biology and p4 medicine: past, present, and future. Rambam Maimonides Medical Journal https://doi.org/10.5041/RMMJ.10112.

Hood, Leroy, und Mauricio Flores. 2012. A personal view on systems medicine and the emergence of proactive P4 medicine: predictive, preventive, personalized and participatory. New Biotechnology 29(6):613-624.

Hood, Leroy, Jennifer C. Lovejoy, und Nathan D. Price. 2015. Integrating big data and actionable health coaching to optimize wellness. BMC Medicine 13(1):4.

Hunter, Peter, Tara Chapman, Peter V. Coveney, et al, 2013. A vision and strategy for the virtual physiological human: 2012 update. Interface Focus https://doi.org/10.1098/rsfs.2013.0004.

Jasanoff, Sheila. 2004. States of knowledge. The co-production of science and social order. New York: Routledge.

Jasanoff, Sheila. 2005. Designs on nature. Science and democracy in Europe and the United States. Princeton, Oxford: Princeton University Press.

Kelleher, John D., und Brendan Tierney. 2018. Data science. Cambridge: MIT Press.

Kirschner, Marc, David Koubi, und Estelle Vincent. 2013. The road map to systems medicine. In Systems Medicine open stakeholder consultation conference. Report. Jülich: CASyM.

Kolany-Raiser, Barbara, Reinhard Heil, Carsten Orwat, und Thomas Hoeren (Hrsg.). 2018. Big Data und Gesellschaft: Eine multidisziplinäre Annäherung. Wiesbaden: Springer VS.

Latour, Bruno. 1994. Science in action. How to follow scientists and engineers through society, 6. Aufl., Cambridge: Harvard University Press.

Latour, Bruno. 2000. Die Hoffnung der Pandora. Frankfurt a. Main: Suhrkamp.

Lemke, Thomas. 2000. Die Regierung der Risiken. Von der Eugenik zur genetischen Gouvernementalität. In Gouvernementalität der Gegenwart. Studien zur Ökonomisierung des Sozialen, Hrsg. Ulrich Bröckling, Susanne Krasmann, und Thomas Lemke, 227-264. Frankfurt a. Main: Suhrkamp.

Lemke, Thomas. 2003. Molekulare Medizin? Anmerkungen zur Ausweitung und Redefinition des Konzepts der genetischen Krankheit. Prokla: Zeitschrift für kritische Sozialwissenschaft 132(3):471-492.

Lemke, Thomas. 2004. Veranlagung und Verantwortung. Genetische Diagnostik zwischen Selbstbestimmung und Schicksal. Bielefeld: transkript.

Lemke, Thomas. 2007. Gouvernementalität und Biopolitik. Wiesbaden: VS.

Lemke, Thomas. 2011. Biopolitics. New York, London: New York University Press.

Leppin, Anja. 2010. Konzepte und Strategien der Prävention. In Lehrbuch Prävention und Gesundheitsförderung, 3. Aufl., Hrsg. Klaus Hurrelmann, Theodor Klotz, und Jochen Haisch, 35-44. Bern: Huber.

Lippman, Abby. 1991. Prenatal genetic testing and screening: Constructing needs and reinforcing inequalities. American Journal of Law \& Medicine 17(1 \& 2):15-50.

Lippman, Abby. 1994. The genetic construction of prenatal testing: choice, consent, or conformity for women? In Women and prenatal testing. Facing the challenges of genetic technology, Hrsg. Karen H. Rothenberg, Elisabeth J. Thomson, 9-34. Columbus: Ohio State University Press.

Lösch, Andreas. 2013. „Vision Assessment“ zu Human-Enhancement-Technologien. Konzeptionelle Überlegungen zu einer Analytik von Visionen im Kontext gesellschaftlicher Kommunikationsprozesse. TATuP - Zeitschrift für Technikfolgenabschätzung in Theorie und Praxis 22(1):9-16.

Lupton, Deborah. 2013. Quantifying the body: monitoring and measuring health in the age of mHealth technologies. Critical Public Health 23(4):393-403.

Lupton, Deborah. 2016. The quantified self: a sociology of self-tracking. Cambridge, Malden: Polity.

Lupton, Deborah, und Annemarie Jutel. 2015. 'It's like having a physician in your pocket!' A critical analysis of self-diagnosis smartphone apps. Social Science \& Medicine 133:128-135.

Maier, Barbara. 2006. Autonomy. On decision-making in prenatal diagnosis. In Prenatal testing: individual decision or distributed action? Science and technology studies, Bd. 48, Hrsg. Bernhard Wieser, Sandra Karner, und Wilhelm Berger, 181-189. München, Wien: Profil.

Miller, Peter, und Nikolas Rose. 2008. Governing the present: administering economic, social and personal life. Cambridge, Malden: Polity.

Moreno, Alvaro, Kepa Ruiz-Mirazo, und Xabier Barandiaran. 2011. The impact of the paradigm of complexity on the foundational frameworks of biology and cognitive science. In Philosophy of complex systems Handbook of the philosophy of science, Bd. 10, Hrsg. Cliff Hooker, 311-333. Amsterdam: Elsevier. 
Nelkin, Dorothy. 1995. Die gesellschaftliche Sprengkraft genetischer Information. In Der Supercode: Die genetische Karte des Menschen, Hrsg. Daniel J. Kevles, 195-209. Frankfurt a. Main: Insel.

Novas, Carlos, und Nikolas Rose. 2000. Genetic risk and the birth of the somatic individual. Economy and Society 29:485-513.

Petersen, Alan, und Deborah Lupton. 1996. The new public health. Health and self in the age of risk. Thousand Oaks: SAGE.

Pinch, Trevor J., und Wiebe E. Bijker. 1984. The social construction of facts and artefacts: or how the sociology of science and the sociology of technology might benefit each other. Social Studies of Science 14(3):399-441.

Prainsack, Barbara. 2017. Personalized medicine. Empowered patients in the 21st century. New York: New York University Press. Biopolitics.

Reif, Maria, und Helmut Baitsch. 1986. Genetische Beratung: Hilfestellung für eine selbstverantwortliche Entscheidung? Heidelberg, Berlin, New York: Springer.

Rheinberger, Hans-Jörg. 1997. Von der Zelle zum Gen. Repräsentationen der Molekularbiologie. In Räume des Wissens: Repräsentation, Codierung, Spur, Hrsg. Hans-Jörg Rheinberger, Michael Hagner, und Bettina Wahring-Schmidt, 265-279. Berlin: Akademie.

Rheinberger, Hans-Jörg. 1999. Reenacting history. Studies in History and Philosophy of Science Part A 30(1):163-166.

Rheinberger, Hans-Jörg, Michael Hagner, und Bettina Wahring-Schmidt (Hrsg.). 1997. Räume des Wissens: Repräsentation, Codierung, Spur. Berlin: Akademie.

Rose, Nikolas. 1999. Powers of freedom: reframing political thought. Cambridge: Cambridge University Press.

Rose, Nikolas. 2007. The politics of life itself: biomedicine, power and subjectivity in the twenty-first century. Princeton: Princeton University Press.

Rose, Nikolas, und Carlos Novas. 2005. Biological citizenship. In Global assemblages. Techology, politics, and ethics as anthropological problems, Hrsg. Aihwa Ong, Stephen Collier, 439-463. Hoboken: Wiley-Blackwell.

Sariola, Salla, und Bob Simpson. 2011. Theorising the 'human subject' in biomedical research: international clinical trials and bioethics discourses in contemporary Sri Lanka. Social Science \& Medicine 73(4):515-521.

Schulz-Schaeffer, Ingo. 2000. Sozialtheorie der Technik. Frankfurt a. Main: Campus.

Selke, Stefan. 2014. Lifelogging: Wie die digitale Selbstvermessung unsere Gesellschaft verändert. Berlin: Econ.

Smith, Merritt Roe, und Leo Marx. 1994. Does technology drive history? The dilemma of technological determinism. Cambridge: MIT Press.

Tian, Q., N.D. Price, und L. Hood. 2012. Systems cancer medicine: towards realization of predictive, preventive, personalized and participatory (P4) medicine. Journal of Internal Medicine 271(2):111-121.

Tutton, Richard. 2014. Genomics and the reimagining of personalized medicine. Farnham: Ashgate.

Vandamme, D., W. Fitzmaurice, B. Kholodenko, und W. Kolch. 2013. Systems medicine: helping us understand the complexity of disease. QJM: Monthly Journal of the Association of Physicians 106(10):891-895.

Vegter, M.W. 2018. Towards precision medicine; a new biomedical cosmology. Medicine, Health Care, and Philosophy 21(4):443-456.

Vogt, Henrik, Bjørn Hofmann, und Linn Getz. 2016. The new holism: P4 systems medicine and the medicalization of health and life itself. Medicine, Health Care, and Philosophy 19(2):307-323.

Wang, Rui-Sheng, Bradley A. Maron, und Joseph Loscalzo. 2015. Systems medicine: evolution of systems biology from bench to bedside. Wiley Interdisciplinary Reviews. Systems Biology and Medicine 7(4):141-161.

WHO. 1948. Preamble to the constitution of the World Health Organization as adopted by the International Health Conference. New York: World Health Organization.

Wolkenhauer, Olaf, und Sara Green. 2013. The search for organizing principles as a cure against reductionism in systems medicine. The FEBS journal 280(23):5938-5948.

Wyatt, Sally. 2008. Technological determinism is dead; long live technological determinism. In The handbook of science and technology studies, Hrsg. Edward J. Hackett, Olga Amsterdamska, Michael Lynch, und Judy Wajcman, 165-180. Cambridge: MIT Press. 
Bernhard Wieser Dr. phil., habilitierte sich an der Alpen-Adria-Universität Klagenfurt für das Fach Wissenschafts- und Technikforschung. Seit 2018 ist er Assoziierter Professor am Institute of Interactive Systems and Data Science der Technischen Universität Graz und betreibt dort Forschung und Lehre im Bereich Wissenschafts- und Technikforschung (Science, Technology and Society). 